\title{
Moving-boundary approximation for curved streamer ionization fronts: Numerical tests
}

\author{
Fabian Brau, ${ }^{1}$ Alejandro Luque, ${ }^{1}$ Benny Davidovitch, ${ }^{2}$ and Ute Ebert ${ }^{1,3}$ \\ ${ }^{1}$ Centrum Wiskunde \& Informatica (CWI), P.O. Box 94079, 1090 GB Amsterdam, The Netherlands \\ ${ }^{2}$ Department of Physics, University of Massachusetts, Amherst, Massachusetts 01003, USA \\ ${ }^{3}$ Department of Physics, Eindhoven University of Technology, P.O. Box 513, 5600MB Eindhoven, The Netherlands \\ (Received 13 January 2009; revised manuscript received 20 April 2009; published 26 June 2009)
}

\begin{abstract}
Recently a moving boundary approximation for the minimal model for negative streamer ionization fronts was extended with effects due to front curvature; this was done through a systematic solvability analysis. A central prediction of this analysis is the existence of a nonvanishing electric field in the streamer interior, whose value is proportional to the front curvature. In this paper we compare this result and other predictions of the solvability analysis with numerical simulations of the minimal model.
\end{abstract}

DOI: 10.1103/PhysRevE.79.066211

PACS number(s): 05.45.-a, 47.54.-r, 52.80.-s

\section{INTRODUCTION}

Streamers characterize the initial stages of electric breakdown in sparks, lightning, and sprite discharges; they occur equally in technical and natural processes [1-3]. They are growing plasma channels that appear when strong electric fields are applied to ionizable matter. The essential features of negative (anode-directed) streamers in a nonattaching gas such as argon or nitrogen can be described by the so-called minimal model [4-14]. This model consists of a set of three coupled partial differential equations for the electron density $\sigma$, the ion density $\rho$, and the electric field E. In dimensionless units the model reads

$$
\begin{gathered}
\partial_{t} \sigma-\nabla \cdot(\sigma \mathbf{E})-D \nabla^{2} \sigma=\sigma|\mathbf{E}| \alpha(|\mathbf{E}|), \\
\partial_{t} \rho=\sigma|\mathbf{E}| \alpha(|\mathbf{E}|), \\
\nabla \cdot \mathbf{E}=\rho-\sigma, \quad \mathbf{E}=-\nabla \phi
\end{gathered}
$$

where $D$ is the electron diffusion coefficient and where

$$
\alpha(|\mathbf{E}|)=e^{-1 /|\mathbf{E}|} .
$$

A general discussion of the physical dimensions for this model can be found, e.g., in $[2,4,5,15]$. The model is based on a continuum approximation with local field-dependent impact ionization reaction. Equations (1) and (2) are the continuity equations for the electrons and the ions, taken as immobile due to their much larger mass, while Eq. (3) is the Coulomb equation for the electric field generated by the space charge $\rho-\sigma$ of electrons and ions. Although discharges in air require extensions of the model, simulation results of negative air streamers frequently resemble the minimal model remarkably well $[15,16]$.

Many simulations [6-13] have shown that streamers form a thin curved space charge layer which separates the ionized interior region, $\Omega^{-}$, from the nonionized exterior region, $\Omega^{+}$. This narrow charged layer (the ionization front) enhances the electric field in $\Omega^{+}$ahead of the front and screens it partially in $\Omega^{-}$. In strong background fields after some transient evolution, the width of the ionization front can be much smaller than its radius of curvature $[12,13,15]$. This separation of scales enables one to consider the front as an infinitesimally thin sharp moving interface $\Gamma(t)$. In Fig. 1, we show a rep- resentative snapshot of net charge density of the minimal model (1)-(3), which shows the separation of scales, and depict the corresponding moving boundary approximation. The original nonlinear dynamics is then replaced by a set of linear field equations (frequently of diffusive or Laplace type) on both sides of $\Gamma(t)$; the regions on both sides of $\Gamma(t)$ are denoted as $\Omega^{+}$and $\Omega^{-}$. The linear fields in these regions are determined by boundary conditions on both sides of the interface, $\Gamma(t)^{+}, \Gamma(t)^{-}$, respectively, and on the outer boundaries [assumed to be located far away from $\Gamma(t)$ ]; the nonlinearity enters through the motion of the boundary. The interface dynamics is typically related to gradients of the Laplacian fields in its vicinity.

In the context of streamer dynamics, the concept of an interfacial approximation was probably first sketched by Sämmer in 1933 [17]; later it was developed further by Lozansky and Firsov in the Russian literature and in English in [18]. They considered the streamer interior, $\Omega^{-}$, as ideally conducting, i.e., the electric potential $\phi$ as constant in the interior. The exterior, $\Omega^{+}$, is nonionized and therefore does not contain space charges; the electric potential here solves

$$
\nabla^{2} \phi=0 \text { in } \Omega^{+} \text {. }
$$

The interface was assumed to move with the local electron drift velocity

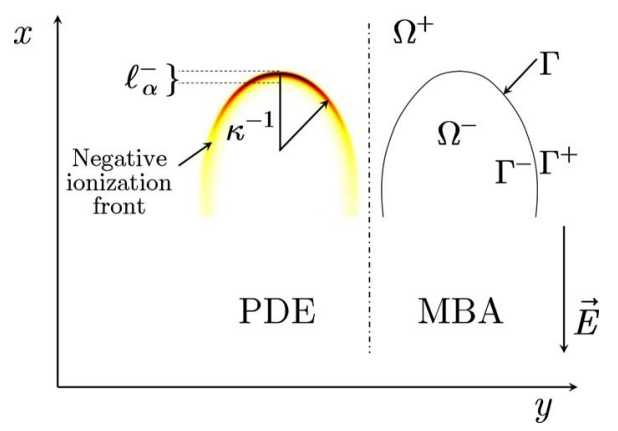

FIG. 1. (Color online) On the left: representative solution (net charge density) of the minimal partial differential equation (PDE) model with curvature $\kappa$ and width $\ell_{\alpha}^{-}$[see Eq. (9)] of the ionization front. The electric field $\vec{E}$ is pointing downward and the negative front propagates upward. On the right: depiction of the corresponding moving boundary approximation (MBA) with the ionized region, $\Omega^{-}$, the nonionized region, $\Omega^{+}$, and the sharp interface, $\Gamma$. 


$$
\mathbf{v}=\nabla \phi^{+} .
$$

Hence, superscripts \pm attached to fields, potential, and densities indicate their limit values as they approach the interface from $\Omega^{+}$and $\Omega^{-}$, respectively. In particular, we denote $\left.\phi^{+} \equiv \phi\right|_{\Gamma^{+}}$and $\left.\phi^{-} \equiv \phi\right|_{\Gamma^{-}}$.

However, this simplest moving boundary approximation is mathematically ill posed; in the context of similar models in fluid dynamics, this is explained, for example, in Ref. [19] and references therein. To resolve this problem, the boundary condition $\phi^{+}-\phi^{-}=0$ was replaced by the regularizing boundary condition

$$
\phi^{+}-\phi^{-}=Q_{0}\left(\hat{\mathbf{n}} \cdot \nabla \phi^{+}\right) \stackrel{\left|\hat{\mathbf{n}} \cdot \nabla \phi^{+}\right| \gg 1}{\rightarrow} \hat{\mathbf{n}} \cdot \nabla \phi^{+},
$$

where $\hat{\mathbf{n}}$ is the unit vector normal to the front pointing toward the nonionized region and $Q_{0}(x)$ is given by Eq. (15). This boundary condition was proposed in [20] and derived in planar front approximation in [21]. The boundary condition accounts for the finite width of the charged layer that leads to a finite variation in the electric potential across the front. The boundary condition in the limit of large electric fields actually turns out to be identical to the "kinetic undercooling" boundary condition that was applied to crystal growth under certain conditions $[22,23]$. Solutions of the model (5)-(7) are discussed in $[20,24]$, and the analysis in [25] shows that boundary condition (7) indeed regularizes the problem. This moving boundary approximation is compared with solutions of the minimal model (1)-(3) in [21,26].

In a recent paper [27], effects associated with curvature of the front were considered. The moving boundary conditions for a slightly curved front dynamics were systematically derived from the original nonlinear field equations (1)-(3), with $D=0$, using the following procedure. A perturbation of a planar front is assumed whose curvature in the direction transverse to the front motion is much smaller than the front width,

$$
\epsilon=\ell_{\alpha}^{-} \kappa \ll 1,
$$

where $\ell_{\alpha}^{-}$and $\kappa$ are the width and the curvature of the front, respectively. The width of the front, $\ell_{\alpha}^{-}$, is taken as the decay length in the ionized region of the net charge density of the planar front with $D=0$ and reads [27]

$$
\ell_{\alpha}^{-}\left(E^{+}\right)=\frac{E^{+}}{\sigma^{-}\left(E^{+}\right)},
$$

where $\sigma^{-}$is the value of the electron density far behind the planar front whose expression is given by Eq. (19) (see, for example, Refs. $[5,27,28])$ and $E^{+}$is the value of the enhanced field in front of the streamer. $\ell_{\alpha}^{-}\left(E^{+}\right)$is a monotonically decreasing function of $E^{+}$and tends to 1 (in our dimensionless units) for $E^{+} \rightarrow+\infty$. The computation is carried out in first order in $\epsilon$. Solvability analysis is then used to connect the perturbed values of the fields ahead and behind the curved front to derive the moving boundary approximation. Similar to other well-studied problems such as solidification dynamics $[29,30]$, this expansion around the planar front solution is asymptotic and does not necessarily converge. However, such a solvability analysis provides a valuable approximation for the nonlinear dynamics of the propagating front as long as $\epsilon$ remains sufficiently small. Furthermore, notice that such an analysis could not be performed on the streamer model with $D>0$ as the fronts are pulled [31-33]. However, the leading edge that pulls the front is diffusive, and it is a physically and mathematically meaningful approximation to neglect electron diffusion in strong fields, where electron motion due to drift dominates over the diffusive motion $[28,34]$. We will come back to this point below.

The complete model derived in Ref. [27] reads

$$
\begin{aligned}
& \nabla^{2} \phi=0 \text { in } \Omega^{+}, \\
& \nabla^{2} \phi=0 \text { in } \Omega^{-},
\end{aligned}
$$

with the moving boundary conditions

$$
\begin{gathered}
\hat{\mathbf{n}} \cdot \nabla \phi^{-}=Q_{2}\left(\hat{\mathbf{n}} \cdot \nabla \phi^{+}\right) \kappa, \\
\phi^{+}-\phi^{-}=Q_{0}\left(\hat{\mathbf{n}} \cdot \nabla \phi^{+}\right)+Q_{1}\left(\hat{\mathbf{n}} \cdot \nabla \phi^{+}\right) \kappa, \\
v_{n}=\hat{\mathbf{n}} \cdot \nabla \phi^{+} .
\end{gathered}
$$

The curvature of the front creates a nonvanishing electric field behind the front [see Eq. (12)], and it also adds a term proportional to the curvature to boundary condition (7) [see Eq. (13)]. Equation (14) is the projection of Eq. (6) onto the normal on the interface, Eq. (10) is the unchanged equation [Eq. (5)], and Eq. (11) will be explained below. The coefficients $Q_{i}$ depend on the electrostatic field ahead of the front and are given by analytic expressions derived from the planar front solution as follows:

$$
\begin{gathered}
Q_{0}(y)=\int_{0}^{y} d z \frac{y-z}{\rho(z, y)}, \\
Q_{1}(y)=-y \int_{0}^{y} d x \frac{(y-x) \alpha(x)}{x \rho(x, y)} \int_{0}^{x} d z \frac{(y-z)}{\rho(z, y)^{2}} \\
-\frac{1}{\sigma^{-}(y)} \int_{0}^{y} d x \frac{\left(y^{2}-x^{2}\right)}{x \rho(x, y)^{2}}\left[\rho(x, y) y-\sigma^{-}(y-x)\right] \\
+\frac{y}{\sigma^{-}(y)} \int_{0}^{y} d x \frac{y-x}{\rho(x, y)}-\frac{y^{3}}{\left[\sigma^{-}(y)\right]^{2}}, \\
Q_{2}(y)=\frac{y^{2}}{\sigma^{-}(y)},
\end{gathered}
$$

where

$$
\begin{gathered}
\rho(x, y)=\int_{|x|}^{y} d \mu \alpha(\mu), \\
\sigma^{-}(y)=\int_{0}^{y} d \mu \alpha(\mu),
\end{gathered}
$$

with $\alpha(x)=e^{-1 / x}$. The quantity $\rho$ is related to the ion density profile of the uniformly translating planar front solution of the minimal model (1)-(3) with $D=0$ (see, for example, Refs. $[5,27,28])$. 
Boundary condition (12) implies that the electric field just behind the ionization front is not completely screened but that it is proportional to the curvature. This implies that the ideal conductivity approximation in the streamer interior $\left(\phi=0\right.$ in $\left.\Omega^{-}\right)$must be relaxed. In Ref. [27], the streamer interior was therefore approximated by assuming charge neutrality $\left(\nabla^{2} \phi=0\right.$ in $\left.\Omega^{-}\right)$. Consequently, boundary condition (12) introduces new physics and leads to a new type of moving boundary problem.

The purpose of this work is to study the validity of this moving boundary model and, for the reason just mentioned, especially the validity of boundary condition (12) by comparing it to numerical solutions of the minimal model (1)-(3). However, it should be noticed that the numerical simulations are performed with a nonvanishing electron diffusion coefficient $D=0.1$ while the moving boundary approximation is derived for $D=0$. On a technical level, this cannot be avoided without major efforts. As stated previously (e.g., in [28,33]), inclusion of diffusion in the moving boundary approximation creates a leading edge of the ionization front that pulls the front along, relaxes algebraically slowly, and ruins a solvability analysis. On the other hand, precisely this diffusion dominated leading edge makes the front smooth and therefore allows the use of the numerical methods developed in [13]; the model with $D=0$ leads to a discontinuity of the electron density that would require quite different numerical methods. However, the limit of large electric field $E^{+}$immediately ahead of the front suppresses the leading edge in a similar manner as the limit of vanishing diffusion $[5,34,35]$. We therefore will see a better agreement between PDE solutions and boundary approximation for larger fields. Furthermore, in this manner we test our boundary conditions on a realistic model and see if our moving boundary model is robust against some changes in the underlying minimal model.

Another relation derived in Ref. [27], which does not appear explicitly in the model (10)-(14), can also be tested against numerical simulations. This is the curvature correction to the value of the electron density behind the front,

$$
\sigma_{\mathrm{back}}=\sigma^{-}\left(\hat{\mathbf{n}} \cdot \boldsymbol{\nabla} \phi^{+}\right)+Q_{3}\left(\hat{\mathbf{n}} \cdot \nabla \phi^{+}\right) \kappa
$$

where

$$
Q_{3}(y)=y \int_{0}^{y} d x \frac{(y-x) \alpha(x)}{x \rho(x, y)} .
$$

The first term in the right-hand side of Eq. $(20), \sigma^{-}\left(\hat{\mathbf{n}} \cdot \boldsymbol{\nabla} \phi^{+}\right)$, is the contribution to the electron density behind the front obtained from a planar front approximation (see Refs. $[5,27,28])$, while the second term takes the effects of the curvature of the front into account.

The paper is organized as follows. In Sec. II, we describe the method used for comparing the moving boundary approximation with the simulation data, and in Sec. III, we describe in detail the results of our comparison concerning boundary conditions (12) and (13) and also Eq. (20).

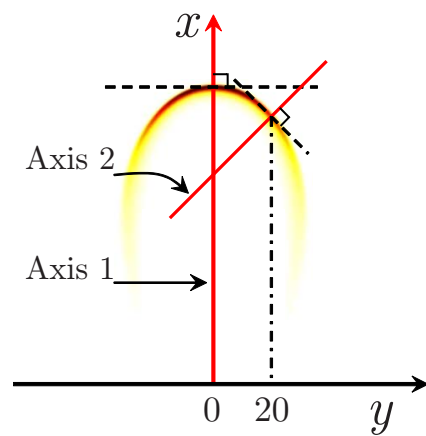

FIG. 2. (Color online) The two axes (solid, red) along which we compare the numerical solutions of the minimal model and the moving boundary approximation. Axis 1 is the symmetry axis of the streamer; it is located at $y=0$. Axis 2 intersects with the front at $y$ $=20$ and it is normal to it.

\section{METHOD FOR COMPARING THE MOVING- BOUNDARY APPROXIMATION WITH SIMULATIONS OF THE MINIMAL MODEL}

In this section, we test boundary conditions (12) and (13) as well as Eq. (20) against results of simulations of the minimal model (1)-(3) in two dimensions. The electric field and the electron density behind the front are essentially constant over a significant interval; therefore it is relatively easy to extract their values from simulation data without introducing significant errors. The comparison with predictions of Eqs. (12) and (20) allows us to test the model with confidence. In contrast, as explained below, due to some arbitrariness of the precise location of $\phi^{+}$in the simulations and since the potential varies significantly over short distances, the comparison between Eq. (13) and the simulations is not quite conclusive.

In order to test our boundary conditions, we need to evaluate the profiles of the net charge density, the electric field and potential, and the electron density along some given axis of the two-dimensional simulated streamer. In this paper we consider a streamer that evolves from initial conditions with a mirror symmetry $y \rightarrow-y$. We perform our analysis along two axes. The first axis is chosen to be the symmetry axis of the streamer located at $y=0$. The second axis is normal to the front and intersects with it at $y=20$; both axes are illustrated in Fig. 2.

\section{A. Numerical simulations}

The minimal PDE model (1)-(3) with $D=0.1[2,4,5]$ is solved numerically in two dimensions on adaptively refined comoving grids with a second-order explicit Runge-Kutta time integration. The algorithm is described in detail in Ref. [13] for three-dimensional cylindrically symmetrical geometries. It is trivially adapted to planar two-dimensional systems, as previously discussed in Refs. [21,26]. The highest spatial resolution in the area around the streamer head was $\Delta x=\Delta y=1 / 4$ for all simulations. The simulation domain was $0 \leq x \leq 2048$ and $-1024 \leq y \leq 1024$. The initial conditions were an electrically neutral Gaussian seed $\sigma(t=0)=\rho(t=0)$ $=A \exp \left[-\left(x^{2}+y^{2}\right) / w^{2}\right]$ of width $w=16$ and height $A=2.4$ $\times 10^{-5}$. We used four different values for the background 


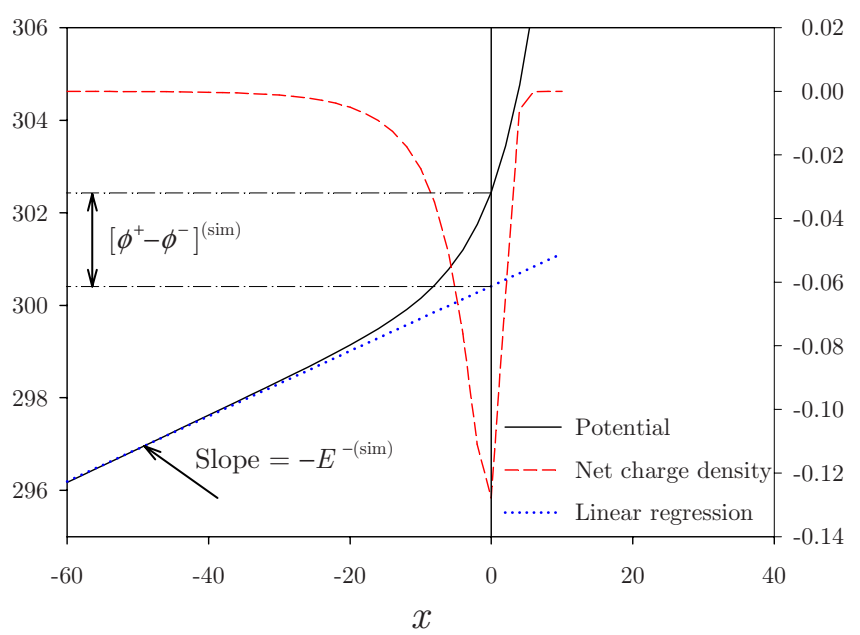

FIG. 3. (Color online) Profiles of the electric potential and of the negative net charge density along the symmetry axis, $y=0$, for $E_{\infty}$ $=-0.5$ and $t=490[\ln (t)=2.69]$. The linear regression for the linear part of the potential is also plotted. The slope of this linear part corresponds to the electric behind the front, $E^{-(\operatorname{sim})}$. The difference between the simulated value of the potential at $x=0$ and the value of the extrapolation of the linear part at the same location gives the jump in the electric potential.

electric field applied between the electrodes, namely, $E_{\infty}$ $=-0.5,-1,-1.5$, and -2 . The simulations are the same as in [21]; for the actual density and field configurations, we refer to the figures in that paper. Notice that the simulations start at $t=0$ but the comparisons between the numerical results and the predictions of the moving boundary approximation are performed from a time $t$ such as the streamer is actually formed, i.e., after the avalanche regime.

\section{B. Extracting relevant quantities from the simulation data}

For each value of $E_{\infty}$, we collected at constant time steps, up to the time of branching, the values of the curvature of the front, of the enhanced electric field (defined as the maximum of the electric field along the axis where the analysis is performed) and the profiles of the electric potential and electron density. These are the ingredients of boundary conditions (12) and (13) and of Eq. (20) that we test in this paper.

To test both relations (12) and (13) using a unique procedure, we consider Eq. (128) in Ref. [27] (with the leading contribution of the planar front added),

$$
\phi(0)-\phi(x)=Q_{0}\left(E^{+}\right)+Q_{1}\left(E^{+}\right) \kappa-Q_{2}\left(E^{+}\right) \kappa x,
$$

where $|x| \gg \ell_{\alpha}^{-}$and where $x=0$ corresponds to the position of the tip of the front, i.e., to the position of the discontinuity line $\Gamma(t)$. This equation predicts that the correction to the potential profile due to curvature is a linear function of the variable $x$ behind the front in an intermediate region between the inner region and the outer region [27]. The slope $Q_{2}\left(E^{+}\right) \kappa$ of linear curve (22) (indicated with linear regression in Fig. 3 ) should be identical to the electric field $E^{-}$behind the front through boundary condition (12); therefore this procedure tests the boundary condition directly. Equation (22) can also be used to test relation (13). Here the position $x=0$ where the potential $\phi(0)$ is evaluated has to be fixed; it is taken as the location of the maximum of the negative net charge density, while $E^{+}$is identified with the maximum of the electric field along the axis along which the analysis is performed (see Fig. 2). The linear regression of the potential, $\phi_{\text {lin }}$, is then extrapolated up to the tip of the front $(x=0)$ and the difference between this value obtained for the potential, $\phi_{\text {lin }}(0)$, and the value of the potential obtained from the simulation at $x=0, \phi(0)$, is compared. Indeed we have

$$
\phi(0)-\phi_{\text {lin }}(0)=Q_{0}\left(E^{+}\right)+Q_{1}\left(E^{+}\right) \kappa,
$$

where $\phi(0)$ can be measured from the simulated potential and $\phi_{\text {lin }}(0)$ is obtained from the linear regression; the procedure is illustrated for $E_{\infty}=-0.5$ and $t=490[\ln (t)=2.69]$ in Fig. 3. We then can compare the simulated potential jump with the theoretical value on the right-hand side of Eq. (23), which corresponds to boundary condition (13).

In order to compute the curvature of the ionization front, we need to define a one-dimensional curve from the diffuse two-dimensional front. For this purpose, we use the following procedure. Let the streamer propagate along the $x$ axis, $y$ being the transverse axis. For a given value of $x$, we locate the position of the maximum of the net charge density along the $y$ axis to get two points (due to mirror symmetry) of the one-dimensional curve. We repeat the procedure for each value $x$ along the streamer length to get the complete onedimensional curve: $y(x)$ indicates the position of the maximal charge density for every $x$. The same procedure was used previously in Ref. [26]. We estimate the curvature $\kappa$ of the front by fitting the section of the curve $y(x)$ around a point $\left[x_{0}, y_{0}=y\left(x_{0}\right)\right]$ with a polynomial $x-x_{0}=a\left(y-y_{0}\right)^{2}+b\left(y-y_{0}\right)$ $+O\left(y^{3}\right)$ and using the standard expression $\kappa=\mid 2 a /(1$ $\left.+b^{2}\right)^{3 / 2}$.

The enhanced field $E^{+(\operatorname{sim})}$ is identified with the maximum of the absolute value of the electric field in the simulations along the axis used to perform the analysis [axis 1 or 2 (see Fig. 2)].

The extraction of $E^{-}$from the simulations, $E^{-(\operatorname{sim})}$, is obtained from the profile of the electric potential along the axis 1 or 2 as already explained above (see also Fig. 3), while its value, obtained within the moving boundary approximation, $E^{-(\mathrm{MBA})}$, is computed using Eq. (12).

The potential behind the front, $\phi^{-}$, is obtained together with $E^{-(\operatorname{sim})}$ since the latter is given by the slope of the linear part of the simulated potential behind the front while the former is given by the intersection of the linear regression with the position of the tip of the front [the position of the discontinuity line $\Gamma(t)]$ that here was chosen to be the maximum of the net charge density.

The potential ahead of the front, $\phi^{+}$, is identified with the value of the potential at the location of the maximum of the net charge density. We also report later in Fig. 9 the values of the potential at two grid points on our finest grid, adjacent to the location chosen to be the discontinuity line, $\Gamma(t)$, of the front.

The electron density behind the negative front, $\sigma_{\text {back }}^{(\operatorname{sim})}$, is obtained from the simulations as $\sigma_{\text {back }}=\frac{1}{2}\left[\sigma\left(x_{\text {back }}\right)+\sigma\left(x_{\text {end }}\right)\right]$, where $x_{\text {back }}$ is the position where the net charge density vanishes. Such point must exist since we start with a neutral 


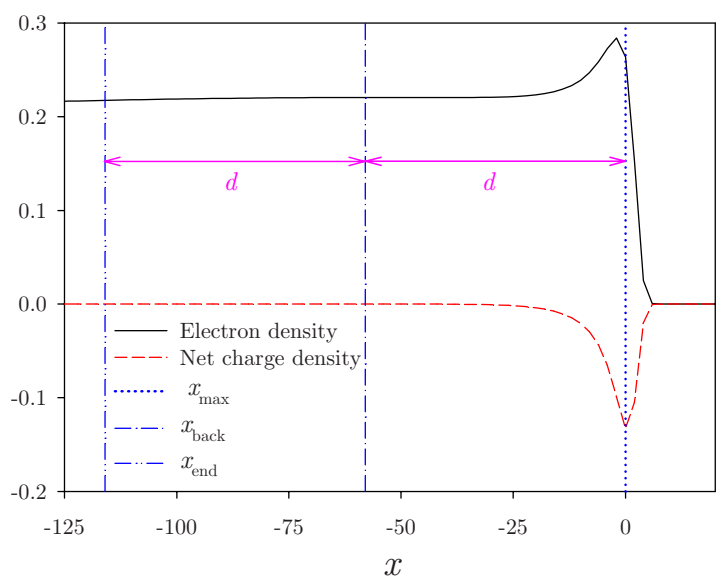

FIG. 4. (Color online) Electron and net charge density profiles for $E_{\infty}=-0.5$ and $t=490[\ln (t)=2.69]$. The position of the maximum of the negative net charge density, $x_{\max }$, and the positions where the charge density vanishes, $x_{\text {back }}$ and $x_{\text {end }}$ (see text), are also indicated. The distance $d$ is equal to $x_{\max }-x_{\text {back }}$.

seed between the electrodes and thus when the streamer forms, positive and negative net charge densities form at the streamer edges and, consequently, the charge density vanishes somewhere in between. The abscissa $x_{\text {end }}$ is defined as $x_{\text {end }}=x_{\max }-2\left(x_{\max }-x_{\text {back }}\right)$, where $x_{\max }$ is the position of the maximum of the net charge density (see Fig. 4). The quantity $\sigma\left(x_{\text {end }}\right)\left[\sigma\left(x_{\text {back }}\right)\right]$ is then the lower (upper) end of the error bars on the value of the electron density behind the negative front. This procedure gives an estimation of the interval of variation in $\sigma$ behind the front. In Fig. 4, we illustrate the procedure for $E_{\infty}=-0.5$ at time $t=490[\ln (t)=2.69]$.

The main source of errors in extracting the relevant quantities from the simulation data are the diffusive nature of the simulated front and hence the nonuniqueness in identifying the interface $\Gamma(t)$. This uncertainty has no influence on the extraction of the quantities $E^{-(\mathrm{sim})}$ and $\sigma_{\text {back }}^{(\text {sim) }}$ from the simulation data since those quantities are evaluated far enough behind the front where they are essentially constant. The error on the slope of the linear part of the potential behind the front, which gives $E^{-(\mathrm{sim})}$, is negligible for our purpose. The interval $\left[x_{\text {end }}, x_{\text {back }}\right]$ where we chose to measure $\sigma_{\text {back }}^{\text {(sim) }}$ is, of course, somewhat arbitrary, but since the electron density is essentially constant behind the front, another procedure would give equivalent results; only the size of the error bars could be slightly different. Consequently the errors on these two quantities are well controlled. The errors for the extracted value $E^{+(\text {sim) }}$ are also negligible for our purpose since this quantity is evaluated on the finest grid $(\Delta x=\Delta y=1 / 4)$ used in the simulations. However, the uncertainty about the exact position of $\Gamma(t)$ affects the extraction of $\phi^{+}$and $\phi^{-}$. Indeed, the location where we chose to evaluate $\phi^{+}$and $\phi^{-}$ on the potential profile is rather arbitrary. Moreover, the potential and the linear regression vary significantly over short distances as shown in Fig. 3. Consequently, the uncertainty of the location of $\phi^{+}$(and thus of $\phi^{-}$) directly influences the results of the comparison between the moving boundary approximation and simulations. However, as explained in Sec. III $C$, the value $\left[\phi^{+}-\phi^{-}\right]^{(\text {sim) }}$ extracted from the simulation

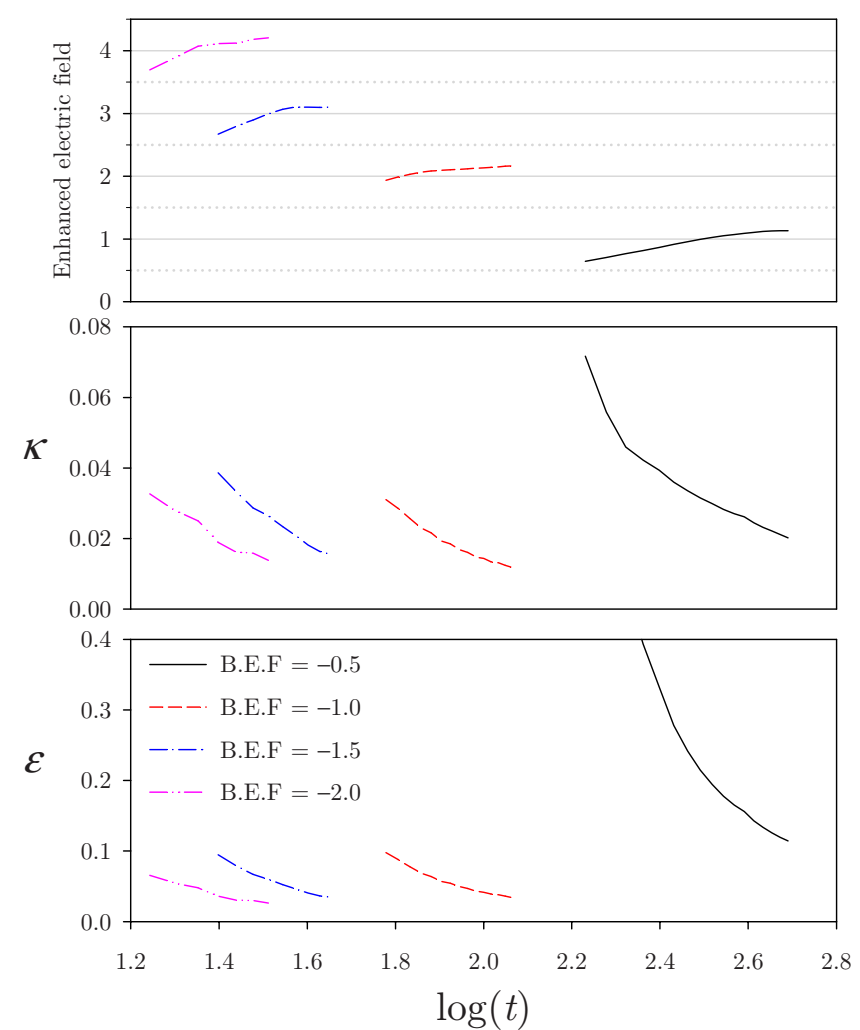

FIG. 5. (Color online) Top: evolution of the absolute value of the maximum of the electric field along the symmetry axis, $y=0$, as a function of time for four values of the background electric field $E_{\infty}$ (B.E.F. on the figure). Middle: evolution of the curvature of the tip of the front as a function of time for the same values of $E_{\infty}$. Bottom: $\epsilon$ as a function of time for the same values of $E_{\infty}$.

data using the procedure described above is an upper bound on the actual value of the potential jump.

\section{Influence of the background electric field}

We expect that the simulation results are better approximated by the moving boundary approximation (12), (13), and (20) when the background electric field, $E_{\infty}$, is large enough. This is so since, as mentioned above, our boundary conditions are derived in the regime $\ell_{\alpha}^{-} \kappa \ll 1$. Formula (9) and simulations indicate that the width of the front is controlled by the value of the enhanced field at the tip of the streamer. Formula (9) derived for planar fronts catches qualitatively the evolution of the front width for a planar interface: the width decreases when the enhanced field increases. Moreover, we notice that in the present simulations (in two dimensions and in a homogeneous electric field) after some initial transients, the value of the enhanced electric field, up to the time of branching, in good approximation is given by (see Fig. 5)

$$
\left|E^{+}\right|=2\left|E_{\infty}\right|+\text { small corrections. }
$$

Consequently, the width of the front is controlled essentially by the background electric field (plus some corrections) and thus for low $E_{\infty}$, where the front width $\ell_{\alpha}^{-}$diverges, one can expect that boundary conditions (12) and (13) and Eq. (20) 


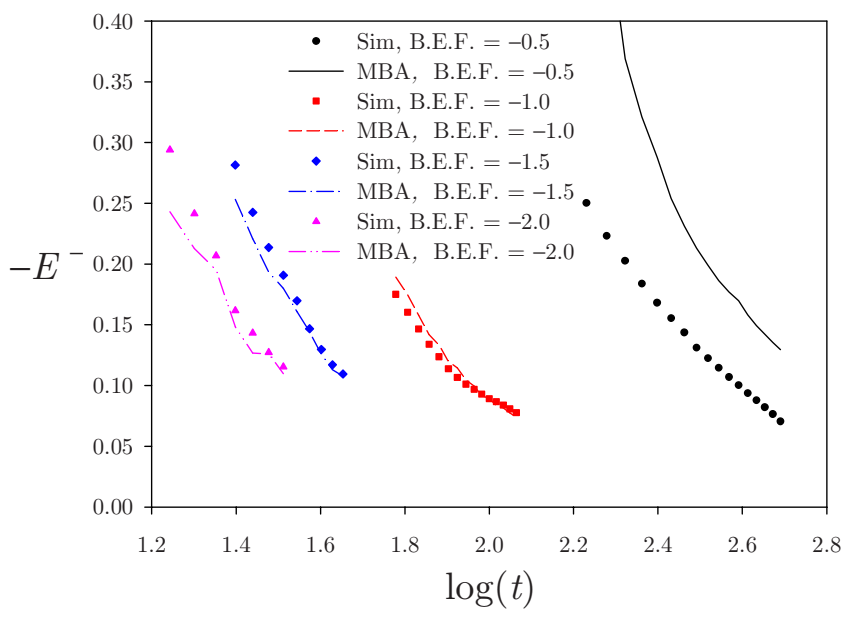

FIG. 6. (Color online) Comparison between the simulated electric field behind negative ionization fronts, $E^{-(\operatorname{sim})}$, and the values, $E^{-(\mathrm{MBA})}$, computed with boundary condition (12) using curvature and enhanced field, $E^{+}$, from the simulations. The comparison is performed for four values of the background electric field (B.E.F. in the figure) and along the symmetry axis of the streamer.

will not approximate the simulations very well. We will show below, however, that for $\left|E_{\infty}\right| \geq 1$, our analytical approximation for the value of the electric field behind the front fits the simulations very well.

For sufficiently large $\left|E_{\infty}\right|$, we expect that relations (12), (13), and (20) approximate the simulations well, and we also expect that the approximation improves with time. Indeed, starting from the initial neutral seed, first an interfacial layer forms and then the value of the enhanced field grows and approaches a plateau value given by Eq. (24). From Eq. (9), this also means that the width of the front decreases during this time. On the other hand, during this process, the curvature of the front also decreases (see Fig. 5). Consequently, for a given $E_{\infty}$, the product $\ell_{\alpha}^{-} \kappa$ decreases during the evolution of the streamer (see Fig. 5). Consequently, since our boundary conditions are derived for $\ell_{\alpha}^{-} \kappa \ll 1$, we expect better agreement between the moving boundary approximation and simulations for time and background electric fields large enough.

This discussion is summarized in the lower panel of Fig. 5, where we show that $\epsilon=\ell_{\alpha}^{-} \kappa$ is a decreasing function of time and of $E_{\infty}$.

\section{RESULTS OF THE COMPARISON}

\section{A. Testing the boundary condition for $E^{-}$}

Following the procedures described in Sec. II B, we extracted the values of $E^{-(\operatorname{sim})}$ from the simulations for four background electric fields: $E_{\infty}=-0.5,-1.0,-1.5$, and -2.0 . These values are then compared with the values, $E^{-(\mathrm{MBA})}$, predicted by Eq. (12) where the curvature, $\kappa$, and the enhanced field, $E^{+}$, are also obtained from the simulations. The results are compared in Fig. 6 for the analysis along the symmetry axis of the streamer (axis 1) and in the top panel of Fig. 10 for the analysis along axis 2. The error bars of $E^{-(\operatorname{sim})}$ are too small to be visible in the figure.
The agreement between the simulations and the moving boundary approximation is rather remarkable except for $E_{\infty}$ $=-0.5$. For this case, the relative errors are always larger than $65 \%$, while for larger background field the errors stay always below 10-12\%. In order to understand why the agreement is less good for $E_{\infty}=-0.5$, we compute $\epsilon$ from Eq. (8). Indeed, we recall that the moving boundary approximation was derived through first-order perturbation theory in $\epsilon$. However, the theory, being linear in $\epsilon$, does not provide an estimation for how small $\epsilon$ should be. Figure 5 shows that for $E_{\infty}=-0.5$, the value of $\epsilon$ stays always above 0.1 . Actually from that figure, we can infer that for $\epsilon \lesssim 0.05$, boundary condition (12) is accurate within $5 \%$ or less.

However, at first sight, $\epsilon$ seems not to be the only control parameter. Indeed, for $E_{\infty}=-1.5$ and $t=25[\ln (t)=1.40]$, we read from Fig. 5 that $\epsilon \simeq 0.10$ and we find that the relative error for $E^{-}$is about $11 \%$ (see Fig. 6), while for $E_{\infty}=-0.5$ and $t=490[\ln (t)=2.69]$, we find that $\epsilon \simeq 0.11$ and that the relative error is about $84 \%$. This means that for the same value of $\epsilon$ we get quite different relative errors for the values of the electric field behind the negative front. However for such a value of $\epsilon$, second-order terms, neglected in the derivation of the moving boundary approximation (10)-(14) (see Ref. [27]), could still play a role. For example, a coefficient associated with $\epsilon^{2}$, which would decrease fast enough with an increase in the enhanced field, may explain why secondorder terms are, in this situation, negligible for larger fields while they still play some role for weaker ones. Secondorder terms could also depend more significantly on the geometry of the streamer by involving a tangential derivative of the curvature. However, without deriving the second-order theory, we cannot draw definitive conclusions on this particular issue.

\section{B. Testing the relation for $\sigma_{\text {back }}$}

Using the procedure described in Sec. II B, we estimated the values of $\sigma_{\text {back }}^{\text {(sim) }}$ from the simulations for the same four background electric fields. These values are then compared with the values, $\sigma_{\text {back }}^{(\mathrm{MBA})}$, predicted by Eq. (20). The results are compared in Fig. 7 when the analysis is performed along axis 1 and in the middle panel of Fig. 10 when the analysis is performed along axis 2 .

The simulation values and those of the moving boundary approximation agree rather well. However, the value of $\sigma_{\text {back }}^{\text {(sim }}$ is slightly underestimated in larger fields. Nevertheless, for $\epsilon \leqq 0.05$, the relative errors are about $10 \%$ or less. Moreover, the curvature correction improves the approximation of the electron density behind the front since the additional term is positive [see Eq. (20)]. In Fig. 8, we compare the effects of the curvature correction for $E_{\infty}=-1.0$.

\section{Testing the boundary condition for $\phi^{+}-\phi^{-}$}

Following the procedures described in Sec. II B, we estimated the values of $\left[\phi^{+}-\phi^{-}\right]^{(\text {sim })}$ from the simulations for the same four background electric fields. These values are then compared with the values, $\left[\phi^{+}-\phi^{-}\right]^{(\mathrm{MBA})}$, predicted by Eq. (13) [or equivalently Eq. (23)]. The results are compared in Fig. 9 when the analysis is performed along axis 1 and in 


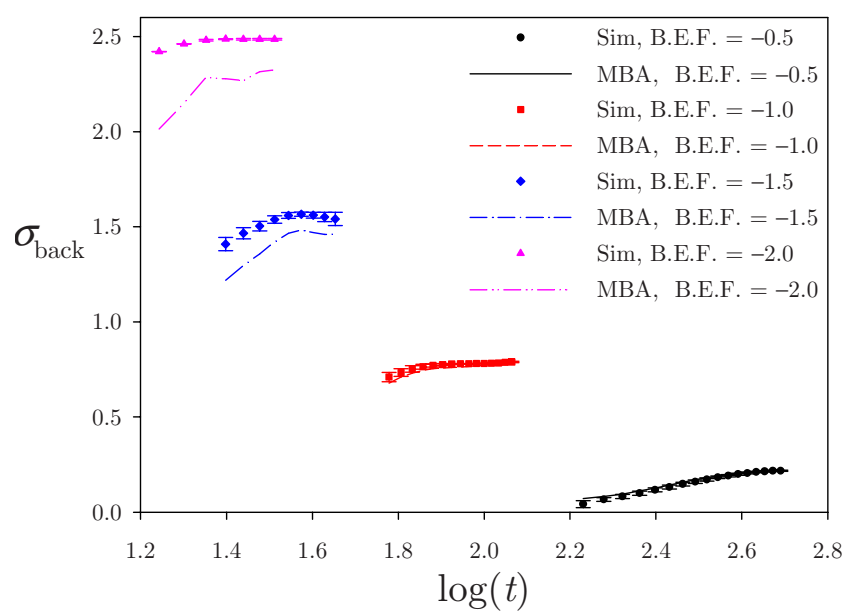

FIG. 7. (Color online) Comparison between the value of the simulated electron densities behind the front, $\sigma_{\text {back }}^{\text {(sim) }}$, and the values, $\sigma_{\text {back }}^{(\mathrm{MBA})}$, computed with Eq. (20) using curvature and enhanced field, $E^{+}$, of the simulations. This comparison is performed for four values of the background electric field (B.E.F. in the figure) and along the symmetry axis of the streamer. The error bars are explained in the text.

the bottom panel of Fig. 10 when the analysis is performed along axis 2. The lower (upper) end of the error bars for the simulation results corresponds to the value of the potential at the grid point just before (after) the position of the maximum of the net charge density on our finest grid. The size of the error bars indicates clearly that indeed the potential varies significantly over quite short distances.

The agreement between the simulation results and the moving boundary approximation for the potential gap [Eq. (13)] is less satisfactory than the excellent agreement demonstrated above for the electric field and charge density [Eqs. (12) and (20)]. Indeed, for $\epsilon \lesssim 0.05$, the relative error is about $20 \%$ or less while for $E^{-}$and the same values of $\epsilon$, the relative error was about $5 \%$ or less. One reason could simply

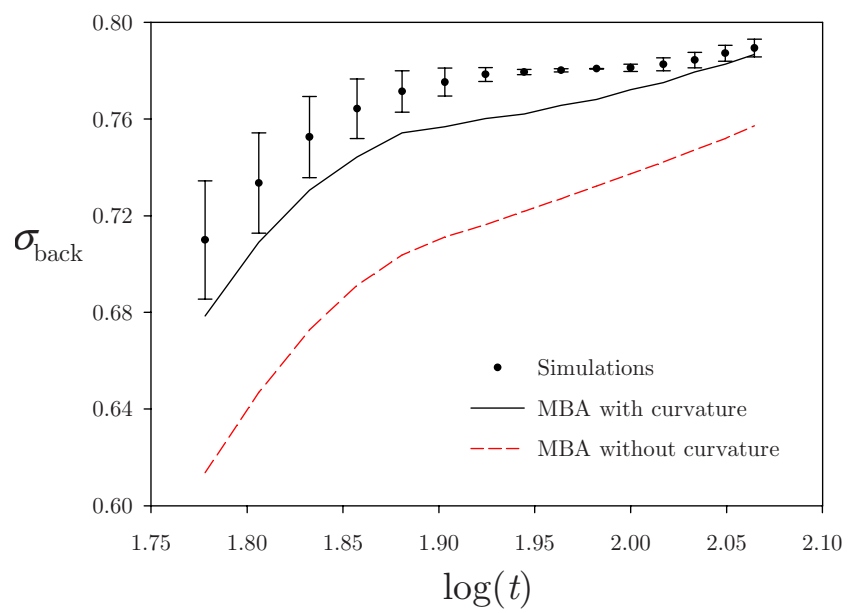

FIG. 8. (Color online) Comparison between the values of the simulated electron density behind the front, $\sigma_{\text {back }}^{(\text {sim })}$, the values, $\sigma_{\text {back }}^{(\mathrm{MBA})}$, computed with Eq. $(20)$, and the values, $\sigma_{\text {back }}^{\text {(MBA) }}$, computed with $\kappa=0$. This comparison is performed for $E_{\infty}=-1.0$ and along the symmetry axis of the streamer.

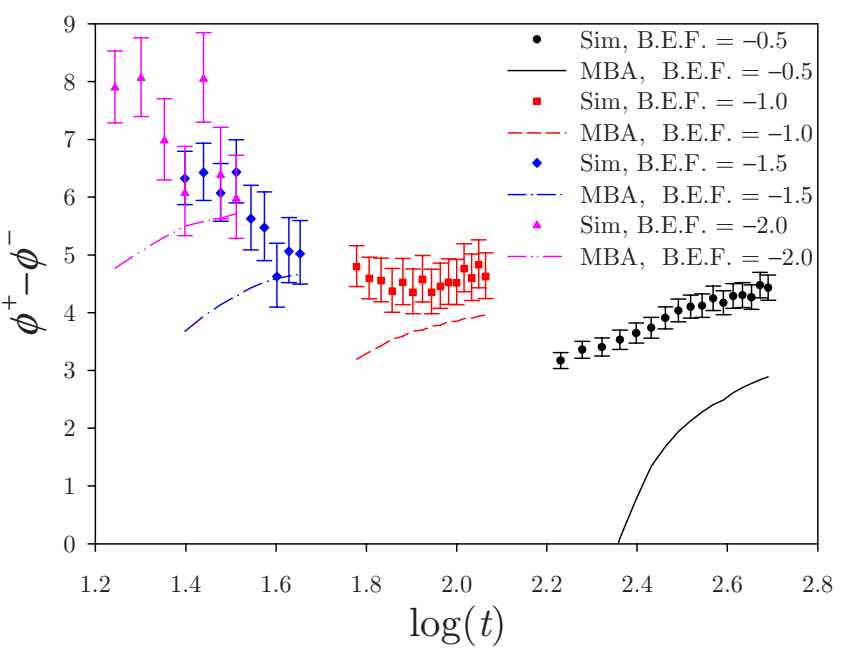

FIG. 9. (Color online) Comparison between the jump of the electric potential across the interface from simulations, $\left[\phi^{+}\right.$ $\left.-\phi^{-}\right]^{(\operatorname{sim})}$, and the values, $\left[\phi^{+}-\phi^{-}\right]^{(\mathrm{MBA})}$, computed with boundary condition (13) where the curvature and the enhanced field, $E^{+}$, are also obtained from the simulations. This comparison is performed for four values of the background electric field (B.E.F. in the figure) and along the symmetry axis of the streamer.

be that the moving boundary approximation works less well for the jump of the electric potential than for $E^{-}$, perhaps due to corrections associated with higher order terms in $\epsilon$. However, another reason is certainly that in this analysis there is one arbitrariness: the precise location for evaluation of $\phi^{+}$. Indeed, as already mentioned above, we choose the location of $\phi^{+}$as the location of the maximum of the negative net charge density. Even if this is a rather natural choice, the actual position of $\phi^{+}$, assumed by the moving boundary approach, could be different. However, because the potential varies significantly over short distances (see Fig. 3 and the size of the error bars on Figs. 9 and 10), the arbitrariness of the location of $\phi^{+}$has certainly a direct influence on the comparison between the moving boundary approximation and the simulations. For example, another possible location for $\phi^{+}$could be the place, $\bar{x}$, such that the amount of negative charge on $x<\bar{x}$ equals the amount of negative charge on $x$ $>\bar{x}$. Since the profile of the net charge density is asymmetric with respect to the position of the maximum (see Fig. 3), $\bar{x}$ would be located before the position of the maximum $(\bar{x}$ $\left.<x_{\max }\right)$ and the jump of the electric potential extracted from the data would be smaller since the potential and its linear regression are increasing functions of $x$. Consequently, the quantity $\left[\phi^{+}-\phi^{-}\right]^{(\operatorname{sim})}$ extracted from the data using our procedure is actually an upper bound on the potential jump assumed in the moving boundary approach.

\section{CONCLUSIONS}

In this paper, we have tested the recently derived moving boundary approximation [27] for negative ionization fronts on simulations of the minimal model (1)-(3).

Our analysis confirmed the validity of two out of the three moving boundary conditions derived in [27], pertaining to 


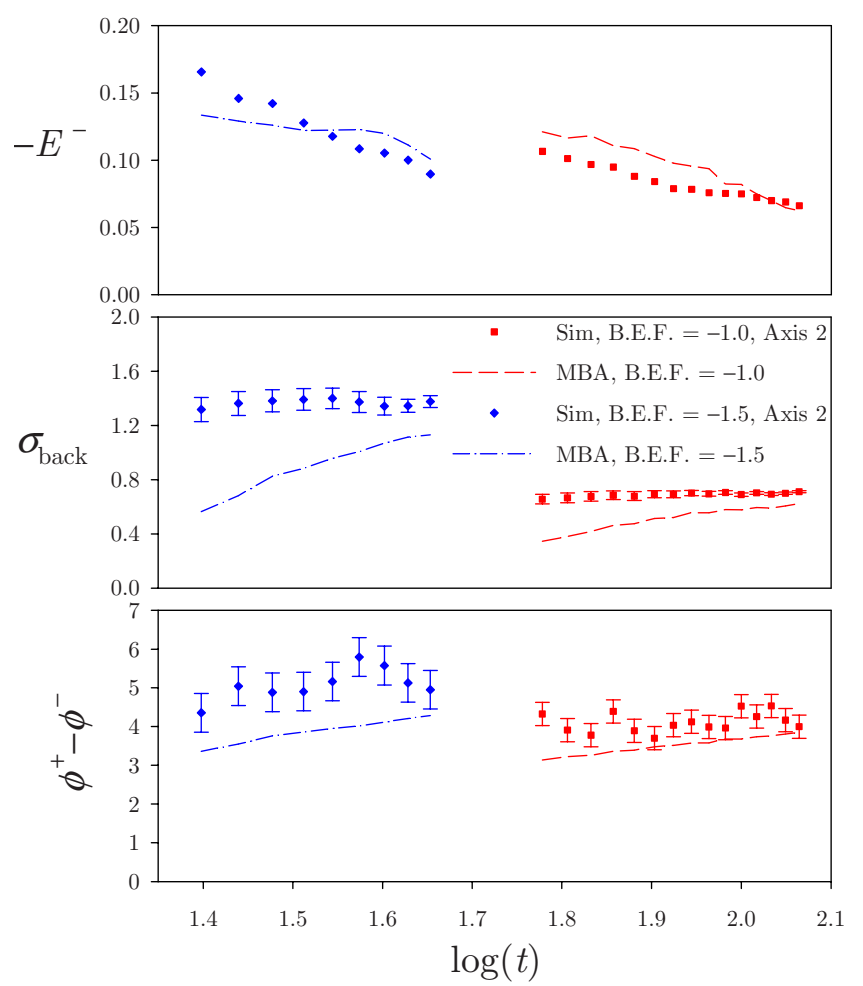

FIG. 10. (Color online) Comparison of moving boundary approximation and simulation along the off-center axis 2 as indicated in Fig. 2 for two values of the background electric field (B.E.F. in the figure). Top: comparison between the simulated electric field behind negative ionization fronts, $E^{-(\operatorname{sim})}$, and the values, $E^{-(\mathrm{MBA})}$, computed with boundary condition (12). Middle: comparison between the value of the simulated electron densities behind the front, $\sigma_{\text {back }}^{(\mathrm{sim})}$, and the values, $\sigma_{\text {back }}^{(\mathrm{MBA})}$, computed with Eq. (20). Bottom: comparison between the jump of the electric potential across the interface from simulations, $\left[\phi^{+}\right.$ $\left.-\phi^{-}\right]^{(\mathrm{sim})}$, and the values, $\left[\phi^{+}-\phi^{-}\right]^{(\mathrm{MBA})}$, computed with boundary condition (13). the curvature dependence of the electrostatic field and the charge density in the ionized region behind the propagating front. We showed that these boundary conditions are satisfied for slightly curved fronts, characterized by a small ratio between the front width $\ell_{\alpha}^{-}$and the radius of curvature $\kappa^{-1}$ of the front. A third boundary condition, concerning the potential jump across the curved front, has not been fully confirmed-a problem that we attribute to the inherent arbitrariness in extracting the appropriate potential values (corresponding to their value at the discontinuity line assumed by the moving boundary approach) from simulations. Further study of the range of validity of this condition will require the development of quantitative tools for such analysis.

The moving boundary approximation improves with growing electric field that coincides with a decreasing contribution of diffusive effects; here we recall from Sec. I that the boundary approximation requires vanishing diffusion while the PDE solution requires a small nonvanishing diffusion coefficient for the analytical and numerical methods presently available for the authors to work.

Finally, the usefulness of the moving boundary approach for analytic and numerical studies of streamer dynamics depends crucially on its capability to describe front dynamics when the ratio $\epsilon$ is not small, as could happen, at least in principle, along some regimes of the propagating front. A progress in studying this important question will require extension of the MBA derived in [27] to such regime and comparison with numerical simulations along the approach developed in this paper.

\section{ACKNOWLEDGMENTS}

The work of F.B. was supported by The Netherlands Organization for Scientific Research (NWO) through Contract No. 633.000.401 within the program "Dynamics of Patterns." The work of A.L. was supported by the Dutch STW Project No. 06501. The work of B.D. in The Netherlands was supported by NWO.
[1] Yu. P. Raizer, Gas Discharge Physics (Springer, Berlin, 1991).

[2] U. Ebert, C. Montijn, T. M. P. Briels, W. Hundsdorfer, B. Meulenbroek, A. Rocco, and E. M. van Veldhuizen, Plasma Sources Sci. Technol. 15, S118 (2006).

[3] U. Ebert and D. D. Sentman, J. Phys. D 41, 230301 (2008).

[4] U. Ebert, W. van Saarloos, and C. Caroli, Phys. Rev. Lett. 77, 4178 (1996).

[5] U. Ebert, W. van Saarloos, and C. Caroli, Phys. Rev. E 55, 1530 (1997).

[6] C. Wu and E. E. Kunhardt, Phys. Rev. A 37, 4396 (1988).

[7] S. K. Dhali and P. F. Williams, Phys. Rev. A 31, 1219 (1985).

[8] S. K. Dhali and P. F. Williams, J. Appl. Phys. 62, 4696 (1987).

[9] P. A. Vitello, B. M. Penetrante, and J. N. Bardsley, Phys. Rev. E 49, 5574 (1994).

[10] M. Arrayás, U. Ebert, and W. Hundsdorfer, Phys. Rev. Lett. 88, 174502 (2002).

[11] A. Rocco, U. Ebert, and W. Hundsdorfer, Phys. Rev. E 66,
035102(R) (2002)

[12] C. Montijn, U. Ebert, and W. Hundsdorfer, Phys. Rev. E 73, 065401(R) (2006).

[13] C. Montijn, W. Hundsdorfer, and U. Ebert, J. Comput. Phys. 219, 801 (2006).

[14] C. Li, W. J. M. Brok, U. Ebert, and J. J. A. M. van der Mullen, J. Appl. Phys. 101, 123305 (2007).

[15] A. Luque, U. Ebert, C. Montijn, and W. Hundsdorfer, Appl. Phys. Lett. 90, 081501 (2007).

[16] A. Luque, V. Ratushnaya, and U. Ebert, J. Phys. D 41, 234005 (2008).

[17] J. J. Sämmer, Z. Phys. 81, 440 (1933).

[18] E. D. Lozansky and O. B. Firsov, J. Phys. D 6, 976 (1973).

[19] S. Howison, SIAM J. Appl. Math. 46, 20 (1986).

[20] B. Meulenbroek, U. Ebert, and L. Schäfer, Phys. Rev. Lett. 95, 195004 (2005).

[21] F. Brau, A. Luque, B. Meulenbroek, U. Ebert, and L. Schäfer, 
Phys. Rev. E 77, 026219 (2008).

[22] H. K. Kuiken, IMA J. Appl. Math. 35, 117 (1985).

[23] S. J. Chapman and J. R. King, J. Eng. Math. 46, 1 (2003), and references therein.

[24] U. Ebert, B. Meulenbroek, and L. Schäfer, SIAM J. Appl. Math. 68, 292 (2007).

[25] S. Tanveer, L. Schäfer, F. Brau, and U. Ebert, Physica D 238 , 888 (2009).

[26] A. Luque, F. Brau, and U. Ebert, Phys. Rev. E 78, 016206 (2008).

[27] F. Brau, B. Davidovitch, and U. Ebert, Phys. Rev. E 78, 056212 (2008).
[28] M. Arrayás and U. Ebert, Phys. Rev. E 69, 036214 (2004).

[29] A. Karma and W. J. Rappel, Phys. Rev. E 57, 4323 (1998).

[30] K. R. Elder, M. Grant, N. Provatas, and J. M. Kosterlitz, Phys. Rev. E 64, 021604 (2001).

[31] U. Ebert and W. van Saarloos, Phys. Rev. Lett. 80, 1650 (1998).

[32] U. Ebert and W. van Saarloos, Physica D 146, 1 (2000).

[33] U. Ebert and W. van Saarloos, Phys. Rep. 337, 139 (2000).

[34] G. Derks, U. Ebert, and B. Meulenbroek, J. Nonlinear Sci. 18, 551 (2008).

[35] M. Arrayás, M. A. Fontelos, and J. L. Trueba, Phys. Rev. Lett. 95, 165001 (2005). 